\title{
Hemogasometria em eqüinos com compactação experimental do cólon maior tratados com sene, fluidoterapia enteral e parenteral
}

\author{
Blood gas analysis in equine with experimental large colon impaction treated with sene, \\ enteral and parenteral fluid therapy
}

\author{
José Dantas Ribeiro Filho ${ }^{I}$ José Mário Girão Abreu ${ }^{\text {II }}$ Geraldo Eleno Silveira Alves ${ }^{\text {III }}$ \\ Waleska de Melo Ferreira Dantas ${ }^{\mathrm{IV}}$
}

\section{RESUMO}

O equilíbrio ácido-base foi estudado em eqüinos com compactação experimental do cólon maior após o uso de solução isotônica poliônica enteral, sene mais hidratação intravenosa e hidratação intravenosa. As amostras sangüíneas foram colhidas de 20 animais, quatro grupos (E8, RL, SE e C) de cinco animais cada. Os animais dos grupos tratados eram portadores de compactação no cólon maior induzida experimentalmente. Eqüinos no grupo E8 receberam solução isotônica poliônica enteral ( $\left.8 \mathrm{~mL} \mathrm{~kg}^{-1} \mathrm{~h}^{-1} 48 \mathrm{~h}^{-1}\right)$; no grupo SE, sene foram administrados na dose de $20 \mathrm{mg} \mathrm{kg}^{-1}$, duas doses de $24 / 24 h$, mais Ringer lactato intravenoso, $10 \mathrm{~mL} \mathrm{kg-1} \mathrm{h}^{-1} 12 \mathrm{~h}^{-1}$, durante dois dias; sendo que o grupo $R L$ recebeu Ringer lactato intravenoso (16mL kg-1 $\left.\mathrm{kg}^{-1} 12 \mathrm{~h}^{-1}\right)$ durante dois dias. O grupo $C$, controle, não foi tratado. Entre os tratamentos testados, a fluidoterapia intravenosa com solução de Ringer lactato (RL) foi o tratamento mais eficiente para a correção das variáveis hemogasométricas em eqüinos com compactação do cólon maior. A solução isotônica poliônica enteral (E8) e sene associada com fluidoterapia intravenosa (SE) foram eficientes, porém em menor grau.

Palavras-chave: eqüino, hemogasometria, fluidoterapia, compactação, cólon maior.

\section{ABSTRACT}

The blood acid-base status was studied in equines when induced large colon impaction was followed by applying an isotonic polionic enteral solution, or sene associated with intravenous fluid therapy, or fluid therapy alone. The blood samples were collected in twenty animals, four groups (E8, RL, $S E$, and $C$ ) of five animals each. Animals of the treated groups had experimentally induced colon impactions. Equines in group E8 received isotonic polionic enteral solution $\left(8 \mathrm{~mL} \mathrm{~kg}^{-1} \mathrm{~h}^{-1} 48\right.$ $\left.h^{-1}\right)$; in group SE, sene was applied at $20 \mathrm{mg} \mathrm{kg}^{-1}$, two doses per animal, at 24-hour intervals, along with the Ringer lactate IV, $10 \mathrm{~mL} \mathrm{~kg}^{-1} \mathrm{~h}^{-1}$, $12 \mathrm{~h}^{-1}$, both during two days; and in the $R L$

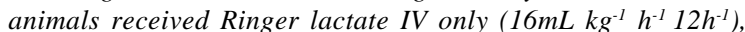
applied during two days. Group $C$ was the non-treated control. Among the tested treatments, the intravenous fluid therapy with Ringer solution (RL) alone was the most efficient to correct the blood gas parameters in horses with large colon impaction. The isotonic polionic enteral solution (E8) and sene associated with intravenous fluid therapy (SE) were also efficient, although less so than Ringer solution alone.

Key words: equine, blood gas analysis, fluid therapy, impaction, large colon.

\section{INTRODUÇÃO}

A homeostase é considerada um dos princípios fundamentais da fisiologia e, dentre os muitos processos que a mantêm, destaca-se a regulação do equilíbrio ácido-base (MUIR \& deMORAIS, 1996). A hemogasometria e a mensuração de eletrólitos são exames laboratoriais importantes para caracterização e avaliação da intensidade dos desequilíbrios hidroeletrolíticos e ácido-base. Estes exames habilitam o veterinário a instituir intervenções terapêuticas apropriadas. Anormalidades eletrolíticas e ácido-base usualmente não definem o diagnóstico, mas certas enfermidades são caracterizadas por predizerem a tendência nesses parâmetros (JOHNSON, 1995).

Em distúrbios clínicos importantes, como diarréia, peritonite, pleurite, disfagia, insuficiência renal, rabdomiólise e cólica, a avaliação laboratorial do

\footnotetext{
IDepartamento de Veterinária, Universidade Federal de Viçosa (DVT/UFV). Av. P. H. Rolfs s/n, Centro, 36570-000, Viçosa, MG, Brasil. E-mail: dantas@ufv.br Autor para correspondência.

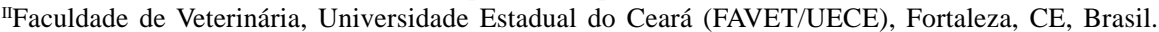

IIIEscola de Veterinária, Universidade Federal de Minas Gerais (EV/UFMG), Belo Horizonte, MG, Brasil.

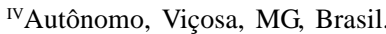


equilíbrio ácido-base e dos eletrólitos deve sempre ser considerada como exames importantes pelo profissional que trabalha com eqüinos (CARLSON, 1997).

Dentre as principais causas de cólica, a compactação do cólon maior em eqüinos é considerada uma das mais importantes na casuística do Hospital Veterinário da Universidade Federal de Viçosa. Compactação é a formação de massa compacta de alimento no lume do trato gastrintestinal e pode ocorrer em qualquer segmento intestinal, mas em eqüinos é particularmente freqüente no intestino grosso, sobretudo no cólon maior (DABAREINER \& WHITE, 1995; THOMASSIAN, 1997). Usualmente os pacientes afetados recuperam-se apenas com a terapia medicamentosa (DORAN, 1993) constituída de controle da dor, hidratação intravenosa ou oral e administração de laxantes (SULLINS, 1990; DABAREINER \& WHITE, 1995).

Um dos sinais mais freqüentes em eqüinos com cólica é a desidratação. A hipovolemia decorrente da desidratação induz à baixa perfusão tecidual, resultando em limitado fornecimento de oxigênio aos tecidos e diminuição na excreção de íons $\mathrm{H}^{+}$pelos rins (ALVES et al., 2005). A hipóxia tecidual aumenta a biossíntese do ácido láctico originário do metabolismo anaeróbico (glicólise), liberando-o mais rapidamente do que ele pode ser oxidado ou reconvertido em glicose ou glicogênio pelo fígado, conforme considerou GOSSET et al. (1987). Esse evento é um dos principais fatores responsáveis pelo aparecimento da acidose metabólica em eqüinos desidratados. Segundo MOORE et al. (1977), a acidose láctica é uma desordem metabólica comum em eqüinos com cólica.

Estudo realizado por LARSEN (1994) demonstrou que $66,7 \%$ dos eqüinos com cólica, forma grave, apresentaram acidose metabólica. Entretanto, NAPPERT \& JOHNSON (2001), em estudo retrospectivo de eqüinos com cólica, observaram que a maioria dos pacientes não apresentou acidose metabólica intensa. Além disso, em alguns desses animais, foi observada alcalose metabólica. Por sua vez, DABAREINER \& WHITE (1995), avaliando eqüinos com compactação do cólon maior, encontram animais com acidemia (pH 7,18) à alcalinemia (pH 7,51) e alcalose respiratória $\left(\mathrm{pCO}_{2} 18 \mathrm{mmHg}\right)$ à acidose respiratória $\left(\mathrm{pCO}_{2}\right.$ 53,2mmHg); entretanto, esses resultados não foram discutidos pelos referidos autores. O presente estudo teve o objetivo de avaliar o equilíbrio ácido-base em eqüinos com compactação experimental do cólon maior e tratados com sene, fluidoterapia enteral e parenteral.

\section{MATERIAL E MÉTODOS}

Utilizaram-se 20 eqüinos hígidos, quatro fêmeas (não-gestantes) e 16 machos (quatro não- castrados e 12 castrados), sem raça definida, com idades entre três e 22 anos, escore corporal de três a quatro (SPEIRS, 1997) e peso corporal médio de 295,52kg. Uma semana antes do experimento, após avaliação clínica, foi feito o controle de endoparasitas (febendazole $^{\mathrm{a}}-7,2 \mathrm{mg} \mathrm{kg} \mathrm{kg}^{-1}$ ) e ectoparasitas (deltametrina ${ }^{\mathrm{b}}$ a 0,025\%). Os animais foram alojados em cocheiras individuais para adaptação à dieta com água e feno de coast cross (Cynodon dactylon) à vontade. A ração concentrada comercial ${ }^{c}$ foi fornecida duas vezes ao dia, em quantidade equivalente a $1 \%$ do peso corpóreo, adicionada de $50 \mathrm{~g} \mathrm{dia}^{-1}$ de suplemento minerald ${ }^{\mathrm{d}}$. Utilizou-se o modelo de compactação no cólon maior, protocolo III, de RIBEIRO FILHO \& ALVES (2002).

Os eqüinos foram aleatoriamente separados em quatro grupos de cinco animais (uma fêmea e quatro machos) - um grupo-controle (sem indução de compactação e sem tratamento) e três grupos tratados, de modo uniforme em relação à idade, ao sexo e ao escore corporal. Ao final das $72 \mathrm{~h}$ da fase de indução da compactação, após confirmar a compactação do cólon maior por exame transretal, iniciaram-se os tratamentos: o grupo enteral (E8) foi tratado com solução isotônica poliônica enteral $\left(\mathrm{NaCl} 5 g+\mathrm{KCl} 1 \mathrm{~g}+\mathrm{NaHCO}_{3} 4 \mathrm{~g}+\right.$ glicose 5g) para completar o volume de $1000 \mathrm{ml}$, administrados em bolus na dose de $8 \mathrm{~mL} \mathrm{~kg}^{-1} \mathrm{~h}^{-1} 48 \mathrm{~h}^{-1}$ da solução via sonda nasogástrica; o grupo sene (SE) recebeu solução isotônica parenteral intravenosa de Ringer lactato ${ }^{\mathrm{e}}\left(10 \mathrm{~mL} \mathrm{~kg}^{-1} \mathrm{~h}^{-1} 12 \mathrm{~h}^{-1}\right)$ associada à sene (VALVERDE et al. 1999) na dose $20 \mathrm{mg} \mathrm{kg}^{-1}$ (uma vez ao dia), diluídos em 1.000ml de água, nos dias 1 e 2 de tratamento; o grupo Ringer lactato (RL) recebeu solução isotônica parenteral intravenosa de Ringer lactato $^{\mathrm{e}}\left(16 \mathrm{~mL} \mathrm{~kg}^{-1} \mathrm{~h}^{-1} 12 \mathrm{~h}^{-1}\right)$, nos dias 1 e 2 de tratamento. Para a análise hemogasométrica, as amostras de sangue foram colhidas anaerobicamente, através de punção da artéria carótida, com agulha 40x9f , em seringas plásticas descartáveis ${ }^{g}$ de $3 \mathrm{~mL}$, previamente heparinizadas e acondicionadas em água com gelo e encaminhadas imediatamente para análise das seguintes variáveis: $\mathrm{pH}$ do sangue arterial - $\mathrm{pH}(\mathrm{a})$, pressão parcial de oxigênio do sangue arterial $-\mathrm{pO}_{2}(\mathrm{a})$, pressão parcial do dióxido de carbono do sangue arterial - $\operatorname{pCO}_{2}(\mathrm{a})$, concentração total do dióxido de carbono no plasma do sangue arterial - $\mathrm{ctCO}_{2}(\mathrm{aP})$, concentração de bicarbonato no plasma do sangue arterial - $\mathrm{CHCO}_{3}^{-}(\mathrm{aP})$, concentração de base titulável do sangue arterial - cBase(a) e saturação de oxigênio do sangue arterial - $\mathrm{sO}_{2}(\mathrm{a})$. Essas análises foram realizadas em aparelho de hemogasometria ${ }^{i}$, com calibração automática, segundo os intervalos: T0i, T24i, T48i e T72i (correspondente à fase de indução da 
compactação), T12t, T24t e T48t (correspondente à fase de tratamento) e T72f (correspondente à fase de avaliação final).

As variáveis foram submetidas ao teste de normalidade e homocedasticidade. Os dados foram submetidos à analise de variância com base em um planejamento de medidas repetidas, ou seja, cada tratamento foi avaliado em vários momentos do acompanhamento, usando-se o programa SAS (1985). Avaliaram-se os efeitos do tratamento, do tempo e a interação tratamento $\times$ tempo. Ao apresentarem significância, as variáveis foram submetidas ao teste de comparações múltiplas de médias LSD. Quando as variáveis não atenderam às premissas, mesmo após as transformações, elas foram avaliadas pelos testes não paramétricos de Kruskal-Wallis (entre tratamentos) e Friedman (tratamento ao longo do tempo) (CONOVER, 1980). Todos os resultados foram considerados significativos quando $\mathrm{P}<0,05$.

\section{RESULTADOS E DISCUSSÃO}

Os resultados dos valores do $\mathrm{pH}(\mathrm{a}), \mathrm{pO}_{2}(\mathrm{a})$, $\mathrm{pCO}_{2}(\mathrm{a}), \mathrm{cHCO}_{3}(\mathrm{aP}), \mathrm{ctCO}_{2}(\mathrm{aP}), \mathrm{cBase}(\mathrm{a})$ e sO $(\mathrm{a}), \mathrm{com}$ as respectivas médias, desvios-padrão, estatística calculada e comentário geral estão expressos na tabela 1 .

Nas primeiras 24h de indução da compactação (T2), observou-se discreto aumento dos valores do $\mathrm{pH}(\mathrm{a})$ nos eqüinos dos grupos $\mathrm{E} 8$, SE e RL $(\mathrm{P}=0,0001)$, que ainda se mantiveram no T3(48hi) nos animais dos grupos SE e RL $(\mathrm{P}=0,0001)$. Este aumento foi ocasionado pela administração da furosemida, uma das substâncias utilizadas no modelo de compactação do cólon maior. A sua administração teve como objetivo aumentar a intensidade da desidratação nos animais. O mecanismo pelo qual a furosemida produz efeito diurético é pela sua ação direta sobre a função tubular renal. Ela inibe a reabsorção de $\mathrm{Na}^{+}$e $\mathrm{Cl}^{-}$no ramo ascendente e espesso da alça de Henle, inibindo o transporte de $\mathrm{Na}^{+}, \mathrm{K}^{+}$e $\mathrm{Cl}^{-}$na membrana luminal. A inibição deste transporte impede a concentração ou diluição do líquido, o qual aumenta a liberação de $\mathrm{Na}^{+}$ e $\mathrm{Cl}^{-}$para o túbulo distal e resulta na produção de volumes aumentados de urina isotônica, instalandose o quadro de hipovolemia, hipocloremia e hipopotassemia, como explicaram BREYER \& JACOBSON (1990). Para corrigir a hipovolemia o organismo aumenta a reabsorção de sódio. Entretanto, para manter a eletroneutralidade, há necessidade de que a reabsorção de sódio no túbulo proximal seja acompanhada pela reabsorção de ânions como o cloreto. Como o paciente está com hipocloremia, maior quantidade de sódio alcança o túbulo distal, onde, sob a ação da aldosterona, estimula a secreção de $\mathrm{H}^{+}$para reter $\mathrm{Na}^{+}$. Porém, a excreção renal de $\mathrm{H}^{+}$é diretamente proporcional à reabsorção de bicarbonato, sobrevindo alcalose metabólica, que, no presente estudo, foi confirmada pelo $\mathrm{CHC0}_{3}^{-}(\mathrm{aP})$ e cBase(a) (Tabela 1), confirmando as descrições de ROSE et al. (1986), FREESTONE et al. (1989) e COSTA(2003).

No final da indução da compactação (T4), os animais dos grupos E8, SE e RL apresentaram diminuição nos valores do $\mathrm{pH}(\mathrm{a})$, principalmente nos animais do grupo $\mathrm{E} 8(\mathrm{P}=0,0001)$, a qual, associada com a diminuição da $\mathrm{CHCO}_{3}^{-}$(aP) e cBase(a), confirmou a acidose metabólica (Tabela 1), que teve como provável origem a produção aumentada de lactato durante a glicólise anaeróbica decorrente da hipovolemia, como foi descrito por JOHNSON (1995). Em estudo retrospectivo, DABAREINER \& WHITE (1995) encontraram, em eqüinos com compactação do cólon maior, acidemia (pH 7,18) e alcalinemia ( $\mathrm{pH} 7,51)$; entretanto, esses resultados não foram discutidos pelos autores. No presente trabalho, durante a fase de tratamento (T5 a T7), decorrente da fluidoterapia, os valores do pH sangüíneo, com exceção do E8, retornaram a índices considerados como de referência (KANEKO et al., 1997). Os valores do pH sangüíneo dos animais do E8 apresentaram-se mais elevados $(\mathrm{pH}$ $7,46)$, fato esse decorrente da presença de bicarbonato de sódio na composição da solução eletrolítica usada nos animais desse tratamento $(\mathrm{P}=0,0001)$.

Na tabela 1, constatam-se valores baixos na $\mathrm{pO}_{2}$ (a) nos animais dos grupos E8, SE e RL, nos T2(24hi) $(\mathrm{P}=0,031)$ e T3(48hi) $(\mathrm{P}=0,033)$. Esses valores baixos foram conseqüência da hipovolemia presente neste período. No T4(72hi), apesar de os pacientes apresentarem o maior grau de desidratação da fase experimental, avaliada pelo tempo de enchimento capilar, hematócrito e proteínas plasmáticas totais, os valores da $\mathrm{pO}_{2}$ (a) elevaram-se $(\mathrm{P}=0,033)$, principalmente no SE. Segundo HOGMAN et al. (1986), quando o pH do sangue atinge determinado grau de acidemia, diminui a afinidade da hemoglobina pelo oxigênio, e se a afinidade de $\mathrm{O}_{2}$ pela hemoglobina diminui, ocorre maior liberação de $\mathrm{O}_{2}$ (efeito Borh), ocasionando aumento da $\mathrm{pO}_{2}$ (a), o que justifica os resultados obtidos nos eqüinos deste estudo. Durante os T6(24ht) e T7(48ht) no grupo E8 e os T5(12ht) e T6(24ht) no grupo $\mathrm{SE}$, houve diminuição dos valores da $\mathrm{pO}_{2}(\mathrm{a})$ (hipoxemia), porém esses valores encontravam-se acima dos valores considerados críticos, ou seja, $60 \mathrm{mmHg}$ (GOMES, 1997). Segundo AINSWORTH \& BILLER (1998), a hipoxemia deve ser considerada quando o valor da $\mathrm{pO}_{2}$ (a) for menor que $85 \mathrm{mmHg}$, enquanto 
Tabela 1 - pH do sangue arterial - pH(a), pressão parcial de oxigênio do sangue arterial - $\mathrm{pO}_{2}$ (a), pressão parcial do dióxido de carbono do sangue arterial - $\mathrm{pCO}_{2}(\mathrm{a})$, concentração total do dióxido de carbono no plasma do sangue arterial - $\mathrm{ctCO}_{2}(\mathrm{aP})$, em eqüinos com compactação experimental do cólon maior tratados com sene, fluidoterapia enteral e parenteral

\begin{tabular}{|c|c|c|c|c|c|c|c|c|c|c|}
\hline Tratamento & T0i & \multicolumn{2}{|c|}{ T24i } & \multicolumn{2}{|c|}{ T48i } & T72i & T12t & $\mathrm{T} 24 \mathrm{t}$ & T48t & T72f \\
\hline \multicolumn{11}{|c|}{$\mathrm{pH}^{\mathrm{a}}$ (média \pm desvio-padrão) } \\
\hline $\mathrm{C}$ & $7,41 \pm 0,02$ & \multicolumn{2}{|c|}{$7,41 \pm 0,02$} & \multicolumn{2}{|c|}{$7,41 \pm 0,02$} & $7,42 \pm 0,01$ & $7,42 \pm 0,02$ & $7,42 \pm 0,02$ & $7,42 \pm 0,01$ & $7,41 \pm 0,01$ \\
\hline E8 & $7,41 \pm 0,02$ & \multicolumn{2}{|c|}{$7,45 \pm 0,02$} & \multicolumn{2}{|c|}{$7,40 \pm 0,02$} & $7,31 \pm 0,04$ & $7,33 \pm 0,03$ & $7,41 \pm 0,05$ & $7,46 \pm 0,02$ & $7,44 \pm 0,04$ \\
\hline SE & $7,40 \pm 0,03$ & \multicolumn{2}{|c|}{$7,44 \pm 0,03$} & \multicolumn{2}{|c|}{$7,43 \pm 0,01$} & $7,38 \pm 0,05$ & $7,40 \pm 0,07$ & $7,42 \pm 0,04$ & $7,43 \pm 0,04$ & $7,42 \pm 0,04$ \\
\hline \multirow[t]{3}{*}{ RL } & $7,41 \pm 0,02$ & \multicolumn{2}{|c|}{$7,44 \pm 0,02$} & \multicolumn{2}{|c|}{$7,44 \pm 0,01$} & $7,, 38 \pm 0,04$ & $7,39 \pm 0,04$ & $7,41 \pm 0,04$ & $7,44 \pm 0,03$ & $7,45 \pm 0,04$ \\
\hline & $\mathrm{C}=\mathrm{E} 8=\mathrm{SE}=\mathrm{RL}$ & $\mathrm{E} 8=\mathrm{SE}=$ & $=\mathrm{RL}>\mathrm{C}$ & $\mathrm{SE}=1$ & $\mathrm{~L}>\mathrm{C}=\mathrm{E} 8$ & $\mathrm{C}>\mathrm{SE}=\mathrm{RL}>\mathrm{C}$ & $\mathrm{C}=\mathrm{SE}=\mathrm{RL}>\mathrm{E} 8$ & $\mathrm{C}=\mathrm{E} 8=\mathrm{SE}=\mathrm{RL}$ & $\begin{array}{l}\text { E8>RL,SE,CR } \\
\mathrm{L}>\mathrm{C}\end{array}$ & $\mathrm{RL}=\mathrm{E} 8>\mathrm{SE}=\mathrm{C}$ \\
\hline & & & & & $\mathrm{pO}_{2}^{\mathrm{b}}$ & (média+desvi & -padrão) & & & \\
\hline $\mathrm{C}$ & $101,2 \pm 9,5$ & $102,8 \pm 7$ & & 101, & $\pm 9,2$ & $103,4 \pm 9,5$ & $97,4 \pm 10,9$ & $104,0 \pm 7,2$ & $97,4 \pm 6,3$ & $99,6 \pm 7,4$ \\
\hline E8 & $97,0 \pm 1,6$ & $88,0 \pm 5$ & & 86,6 & 5,5 & $96,4 \pm 5,0$ & $91,0 \pm 9,7$ & $83,6 \pm 12,7$ & $81,6 \pm 11,4$ & $86,0 \pm 9,7$ \\
\hline SE & $102,8 \pm 8,1$ & $92,5 \pm 6$ & & 85,3 & 6,5 & $101,0 \pm 7,2$ & $82,5 \pm 12,8$ & $83,8 \pm 6,4$ & $93,0 \pm 10,6$ & $93,0 \pm 8,4$ \\
\hline RL & $105,8 \pm 5,5$ & $89,2 \pm 9$ & & 84,4 & 11,2 & $91,0 \pm 3,9$ & $108,4 \pm 7,2$ & $99,4 \pm 10,2$ & $96,6 \pm 11,0$ & $106,0 \pm 15,7$ \\
\hline & $\mathrm{C}=\mathrm{E} 8=\mathrm{SE}=\mathrm{RL}$ & $\mathrm{C}>\mathrm{E} 8=$ & $\mathrm{SE}=\mathrm{RL}$ & $\mathrm{C}>\mathrm{E}$ & $=\mathrm{SE}=\mathrm{RL}$ & $\mathrm{C}>\mathrm{RL}$ & $\mathrm{RL}>\mathrm{SE}$ & * & * & $*$ \\
\hline & & & & & $\mathrm{pCO}_{2}$ & ) $)^{\mathrm{a}}$ (média \pm & vio-padrão) & & & \\
\hline $\mathrm{C}$ & $40,8 \pm 3,7$ & $37,2,6 \pm$ & 2,2 & 38,8 & 2,6 & $39,0 \pm 3,9$ & $39,6 \pm 2,1$ & $39,8 \pm 3,4$ & $39,0 \pm 1,2$ & $39,6 \pm 3,7$ \\
\hline E8 & $42,0 \pm 2,6$ & $44,6 \pm 2$ & & 45,4 & 1,5 & $33,8 \pm 7,4$ & $34,2 \pm 3,6$ & $38,4 \pm 15,4$ & $39,8 \pm 5,7$ & $45,0 \pm 3,2$ \\
\hline SE & $39,5 \pm 4,3$ & $41,5 \pm 1$ & & 44,8 & 3,5 & $35,5 \pm 1,7$ & $36,3 \pm 1,7$ & $39,5 \pm 5,5$ & $36,0 \pm 1,4$ & $37,3 \pm 3,9$ \\
\hline RL & $42,2 \pm 1,9$ & $44,2 \pm 1$ & & 43,4 & & $37,2 \pm 4,5$ & $36,6 \pm 3,9$ & $36,6 \pm 3,8$ & $41,0 \pm 2,9$ & $37,0 \pm 0,7$ \\
\hline & $\mathrm{C}=\mathrm{E} 8=\mathrm{SE}=\mathrm{RL}$ & $\mathrm{E} 8=\mathrm{RL}$ & & $\mathrm{E} 8=$ & $E=R L>C$ & $\mathrm{C}>\mathrm{E} 8$ & $\mathrm{C}>\mathrm{E} 8$ & $\begin{array}{l}\mathrm{C}=\mathrm{E} 8=\mathrm{SE}= \\
\mathrm{RL}\end{array}$ & $\mathrm{RL}>\mathrm{SE}$ & $\mathrm{E} 8>\mathrm{C}=\mathrm{E} 8=\mathrm{RL}$ \\
\hline & & & & & $\mathrm{ctCO}_{2}(\mathrm{a}$ & $\mathrm{P})^{\mathrm{a}}$ (média+ & o-padrão) & & & \\
\hline $\mathrm{C}$ & $57,2 \pm 5,0 \quad 54,6$ & $\pm 4,6$ & $57,0 \pm 2$ & & $55,6 \pm 4$ & $59,8 \pm$ & $56,4 \pm 3,9$ & $57,2 \pm 2$ & $57,6 \pm$ & $\pm 4,1$ \\
\hline E8 & $60,, 2 \pm 5,5 \quad 70,6$ & $\pm 5,9$ & $65,6 \pm 4$ & & $38,8 \pm 10$ & $41,4 \pm$ & $55,8 \pm 11$ & $60,0 \pm 1$ & $65,4 \pm$ & $\pm 15,8$ \\
\hline SE & $56,5 \pm 4,5 \quad 63,8$ & $3 \pm 3,3$ & $67,8 \pm 5$ & & $47,3 \pm 3$ & $52,0 \pm$ & $59,5 \pm 14$ & $53,3 \pm 3$ & $55,0 \pm$ & $\pm 6,1$ \\
\hline RL & $62,0 \pm 2,0 \quad 68,6$ & $\pm 3,7$ & $67,2 \pm 3$ & & $51,4 \pm 10$ & $50,2 \pm 0$ & $54,4 \pm 10$ & $62,6 \pm 3$ & $59,0 \pm$ & $\pm 4,4$ \\
\hline & $\mathrm{C}=\mathrm{E} 8=\mathrm{SE} \quad \mathrm{E} 8=$ & $=\mathrm{SE}=\mathrm{RL}$ & $\mathrm{SE}=\mathrm{RI}$ & & $\mathrm{C}=\mathrm{RL}>\mathrm{I}$ & $\mathrm{C}>\mathrm{E} 8$ & $\mathrm{C}=\mathrm{E} 8=\mathrm{S}$ & $\mathrm{C}=\mathrm{E} 8=$ & $E 8>S$ & \\
\hline & & & & & & & RL & RL & & \\
\hline
\end{tabular}

T0i-T72i: período de indução de compactação.

T12t-T48t: período de tratamento.

T72t: período final de tratamento.

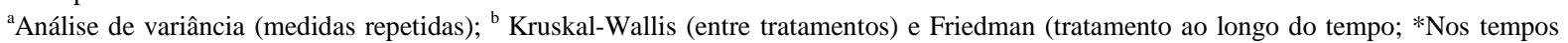
24t, 48t e 72f, os tratamentos não foram comparados, pois um animal do grupo SE morreu.

$\mathrm{C}=$ controle; $\mathrm{E} 8=$ solução isotônica poliônica enteral $\left(\mathrm{NaCl} 5 \mathrm{~g}+\mathrm{KCl} 1 \mathrm{~g}+\mathrm{NaHCO}_{3} 4 \mathrm{~g}+\mathrm{glicose} 5 \mathrm{~g}-\mathrm{qsq} 1000 \mathrm{ml}\right)$, dose de $8 \mathrm{~mL} \mathrm{~kg}^{-1} \mathrm{~h}^{-1} 48 \mathrm{~h}{ }^{-1}$ na sonda nasogástrica; $\mathrm{SE}=$ solução isotônica intravenosa de Ringer lactato $\left(10 \mathrm{~mL} \mathrm{~kg}{ }^{-1} \mathrm{~h}^{-1} 12 \mathrm{~h}^{-1}\right)$ associada à sene, dose de $20 \mathrm{mg} \mathrm{kg}$; $\mathrm{RL}=$ solução isotônica de Ringer lactato $\left(16 \mathrm{~mL} \mathrm{~kg}^{-1} \mathrm{~h}^{-1} 12 \mathrm{~h}^{-1}\right)$.

BAILEY \& PABLO(1998) consideram-na quando a $\mathrm{pO}_{2}$ (a) for menor que $60 \mathrm{mmHg}$. No presente estudo, a hipoxemia nos pacientes dos grupos E8 e SE possivelmente foi ocasionada por processo inflamatório pulmonar, detectado em três animais, dois no grupo E8 e um no SE, o que está de acordo com os relatos de GOMES (1997), que citou como causa da diminuição da $\mathrm{pO}_{2}$ os processos inflamatórios pulmonares. A possível causa do processo inflamatório pulmonar foi o estresse decorrente das fases de indução (T1 a T4) e tratamento (T5 a T7). O estresse no paciente com dor desencadeia, via sistema neuroendócrino, aumento na concentração de cortisol. E, como citou WARNER(2002), o aumento na concentração do cortisol sérico tem papel importante no desenvolvimento da doença respiratória em eqüinos adultos.
Observou-se no T2(24hi), nos grupos E8 e RL, e no T3(48hi), nos grupos E8, SE e RL, aumento da $\mathrm{pCO}_{2}(\mathrm{a})(\mathrm{P}=0,0001)$. Apesar de não poder ser considerada acidose respiratória, pois, segundo KANEKO et al. (1997), somente valores maiores que 46 mmHg indicam acidose respiratória, os valores obtidos estão próximos aos citados pelos autores, podendo ser considerados anormais (Tabela 1). Corroborando essa afirmação, havia alcalose metabólica confirmada pela $\mathrm{CHCO}_{3}^{-}(\mathrm{aP})$ e cBase(a) nos referidos grupos e tempos (Tabela 1). A acidose respiratória geralmente ocorre como mecanismo de compensação da alcalose metabólica ou como alteração primária nas afeç̧ões pulmonares (JONHSON, 1995). Como nos animais havia 
sido administrada furosemida, que é reconhecidamente indutora de alcalose metabólica, acredita-se que a acidose respiratória presente nos animais nesses períodos fosse secundária ou compensatória à alcalose metabólica.

Durante o T4(72hi) e o T5(12ht) ocorreu diminuição dos valores da $\mathrm{pCO}_{2}$ (a) nos animais dos grupos E8, SE e RL ( $\mathrm{P}=0,0001)$, caracterizando a presença de alcalose respiratória, confirmada pela citação de KANEKO et al. (1997), que consideraram sua ocorrência quando os valores da $\mathrm{pCO}_{2}$ forem inferiores a 38mmHg. Corroborando a presença da alcalose respiratória, havia acidose metabólica, diminuição da cBase(aP) (Tabela 1). Segundo JONHSON (1995), a alcalose respiratória geralmente ocorre como mecanismo de compensação ou como alteração primária na hipoxemia, dor e endotoxemia. Ao avaliar eqüinos com compactação do cólon maior, casuística natural, DABAREINER \& WHITE (1995) também encontraram pacientes com alcalose respiratória ( $\mathrm{pCO}_{2} 18 \mathrm{mmHg}$ ) e com acidose respiratória ( $\mathrm{pCO}_{2}$ 53,2mmHg); entretanto, os resultados não foram discutidos pelos referidos autores.

Nos T7(48ht) e T8(72hf), os eqüinos dos grupos SE, e nos T6(24ht) e T8(72hf), os do grupo RL apresentaram alcalose respiratória sem envolvimento metabólico, o que indica que a alteração era de origem primária. No T8(72hf), os animais do grupo E8 apresentaram discreto aumento da $\mathrm{pCO}_{2}(\mathrm{a})$, decorrente do aparecimento de dois pacientes com broncopneumonia, a qual foi ocasionada, possivelmente, pelo estresse decorrente das fases de indução e tratamento da compactação, como explicado anteriormente.

A $\operatorname{ctCO}_{2}(\mathrm{aP})$ é a expressão da reserva alcalina, sendo o bicarbornato o seu principal componente. A diminuição na sua concentração é indicativa de acidose metabólica, enquanto o seu aumento é indicativo de alcalose metabólica (DAVENPORT, 1973; ANDREWS \& GRINDEM, 2000). Nos grupos E8, SE e RL, a cHCO- ${ }_{3}^{-}(\mathrm{aP})$, a ctCO $(\mathrm{aP})$ e a cBase(a) tiveram comportamento semelhante (Tabela 2). Nos T2(24hi) e T3(48hi), houve discreto, mas significativo, aumento nas suas concentrações $(\mathrm{P}=0,0001)$, demonstrando quadro de alcalose metabólica originária da ação da furosemida, mecanismo já explicado anteriormente.

Nos T4 e T5 (final da fase de indução da compactação e início do tratamento), em resposta à presença da hipovolemia, instalou-se acidose metabólica, representada pela diminuição dos valores das referidas variáveis nos animais dos grupos E8, SE e RL. Entretanto, com o decorrer do tratamento, a acidose metabólica foi controlada, fato indicado pelo retorno a valores semelhantes ao T1(0hi). Nos animais do grupo E8, no T8(72hf), observou-se aumento nas concentrações de $\mathrm{cHCO}_{3}^{-}(\mathrm{aP}), \mathrm{ctCO}_{2}(\mathrm{aP})$ e cBase(a) $(\mathrm{P}=0,0001)$, indicando a presença de alcalose metabólica. Este evento possivelmente foi ocasionado pelo mecanismo de compensação em resposta à acidose respiratória, como descreveram CORLEY \& MARR (1998), associado também à composição da solução enteral utilizada nos animais desse grupo.

$\mathrm{O}$ valor de referência para a $\mathrm{pO}_{2}$ (a) é de 90 a 100mmHg (BAILEY \& PABLO, 1998), o que corresponde a $\mathrm{sO}_{2}$ (a) de 97 a 97,5\% (TERZI, 1992). Saturação de $\mathrm{O}_{2}$ de $96 \%$ corresponde a $\mathrm{pO}_{2}$ (a) de 80mmHg, o que no homem significa situação clínica grave (GOMES, 1997). Valores abaixo de 96\% de saturação de $\mathrm{O}_{2}$ foram encontrados nos animais dos grupos SE no T5(12ht) (95,0 $\pm 1,4 \%)$ e nos do RL no T3(48hi) (95,8 $\pm 1,6 \%)$, decorrentes da hipoxemia oriunda da hipovolemia presente nos animais nesses períodos. No entanto, ocorreu recuperação dos pacientes ao longo do tempo, com reposição da volemia em ambos os grupos.

Os eqüinos do grupo E8 apresentaram valores de saturação abaixo de $96 \%$ a partir do T5(12ht) até T8(72hf), no qual atingiram o valor de $94,4 \pm 2,7 \%$ (este valor de saturação corresponde a $\mathrm{pO}_{2}$ (a) de 69,4 a 74,2 mmHg), que, segundo GOMES (1997), representa uma situação clínica extremamente grave no homem. Esse achado foi ocasionado principalmente por dois animais, que desenvolveram hipoxemia em conseqüência da broncopneumonia. Observou-se diminuição discreta da $\mathrm{sO}_{2}$ nesse período (T5-12ht a T8-72hf) nos animais do grupo SE, o qual apresentou um animal com broncopneumonia. Possivelmente, se tivesse ocorrido o aparecimento de broncopneumonia na mesma proporção do grupo E8, seriam obtidos resultados semelhantes nos dois grupos (Tabela 2).

\section{CONCLUSÕES}

A fluidoterapia intravenosa com solução de Ringer lactado é o tratamento mais eficiente para correção das variáveis hemogasométricas em eqüinos com compactação induzida do cólon maior, enquanto a solução isotônica poliônica enteral (E8) e sene associada com fluidoterapia intravenosa (SE) são eficientes, porém em menor grau. 
Tabela 2 - Concentração de bicarbonato no plasma do sangue arterial - $\mathrm{cHCO}_{3}^{-}(\mathrm{aP})$, concentração de base titulável do sangue arterial cBase(a) e saturação de oxigênio do sangue arterial - $\mathrm{sO}_{2}$ (a) em eqüinos com compactação experimental do cólon maior tratados com sene, fluidoterapia enteral e parenteral.

\begin{tabular}{|c|c|c|c|c|c|c|c|c|}
\hline Tratamento & T0i & $\mathrm{T} 24 \mathrm{i}$ & T48i & $\mathrm{T} 72 \mathrm{i}$ & $\mathrm{T} 12 \mathrm{t}$ & $\mathrm{T} 24 \mathrm{t}$ & T48t & $\mathrm{T} 72 \mathrm{f}$ \\
\hline & \multicolumn{8}{|c|}{$\mathrm{cHCO}_{3}^{-}(\mathrm{aP})^{\mathrm{a}}$ (média \pm desvio-padrão) } \\
\hline $\mathrm{C}$ & $24,8 \pm 1,8$ & $24,2 \pm 0,8$ & $24,8 \pm 0,8$ & $24,4 \pm 1,3$ & $26,2, \pm 0,5$ & $25,0 \pm 0,7$ & $24,6 \pm 0,9$ & $25,4 \pm 0,9$ \\
\hline E8 & $26,0 \pm 2,4$ & $30,0 \pm 2,7$ & $27,8 \pm 1,9$ & $16,4 \pm 4,8$ & $17,4 \pm 3,0$ & $23,8 \pm 5,2$ & $27,8 \pm 5,0$ & $29,6 \pm 3,2$ \\
\hline SE & $24,3 \pm 1,0$ & $27,0 \pm 1,2$ & $29,0 \pm 2,5$ & $20,0 \pm 1,4$ & $22,3 \pm 4,4$ & $25,5 \pm 6,1$ & $22,8 \pm 1,3$ & $23,3 \pm 2,5$ \\
\hline \multirow[t]{3}{*}{ RL } & $26,4 \pm 1,1$ & $29,4 \pm 1,7$ & $28,8 \pm 1,3$ & $21,8 \pm 4,2$ & $21,8 \pm 4,2$ & $23,0 \pm 4,7$ & $26,4 \pm 1,8$ & $25,2 \pm 2,2$ \\
\hline & $\begin{array}{l}\mathrm{C}=\mathrm{E} 8=\mathrm{SE}=\mathrm{R} \\
\mathrm{L}\end{array}$ & $\mathrm{E} 8=\mathrm{RL}>\mathrm{C}$ & $\mathrm{SE}=\mathrm{RL}>\mathrm{C}$ & $\begin{array}{l}\text { C>SE,E8RL } \\
>\text { E8 }\end{array}$ & $\begin{array}{l}\mathrm{C}>\mathrm{SE}=\mathrm{RL}>\mathrm{E} \\
8\end{array}$ & $\begin{array}{l}\mathrm{C}=\mathrm{E} 8=\mathrm{SE}=\mathrm{R} \\
\mathrm{L}\end{array}$ & $\mathrm{E} 8>\mathrm{SE}$ & $\begin{array}{l}\mathrm{E} 8>\mathrm{C}=\mathrm{SE}=\mathrm{R} \\
\mathrm{L}\end{array}$ \\
\hline & \multicolumn{8}{|c|}{ cBase(a) (média+desvio-padrão) } \\
\hline $\mathrm{C}$ & $0,4 \pm 1,5$ & $0,2 \pm 0,8$ & $0,2 \pm 0,5$ & $0,8 \pm 1,6$ & $1,8 \pm 1,1$ & $0,8 \pm 0,5$ & $0,8 \pm 0,8$ & $1,0 \pm 1,0$ \\
\hline E8 & $1,6,0 \pm 2,2$ & $6,0 \pm 0,8$ & $3,4 \pm 2,1$ & $-8,6 \pm 4,8$ & $-6,6 \pm 3,0$ & $0,2 \pm 5,1$ & $4,0 \pm 4,7$ & $4,8 \pm 3,7$ \\
\hline SE & $0,3 \pm 1,0$ & $3,3 \pm 1,5$ & $4,5 \pm 1,9$ & $-4,0 \pm 2,2$ & $-1,8 \pm 5,0$ & $1,3 \pm 5,9$ & $-0,5 \pm 2,1$ & $0,0 \pm 2,8$ \\
\hline \multirow[t]{3}{*}{$\mathrm{RL}$} & $2,2 \pm 0,8$ & $5,6 \pm 1,7$ & $4,6 \pm 1,1$ & $-2,0 \pm 4,1$ & $-2,8 \pm 3,3$ & $-0,8 \pm 4,7$ & $3,2 \pm 1,6$ & $2,2 \pm 2,6$ \\
\hline & $\begin{array}{l}\mathrm{C}=\mathrm{E} 8=\mathrm{SE}=\mathrm{R} \\
\mathrm{L}\end{array}$ & $\mathrm{E} 8=\mathrm{RL}>\mathrm{C}$ & $\mathrm{SE}=\mathrm{RL}>\mathrm{C}$ & $\begin{array}{l}\mathrm{C}>\mathrm{SE}, \mathrm{E} 8 \mathrm{RL} \\
>\mathrm{E} 8\end{array}$ & $\begin{array}{l}\mathrm{C}>\mathrm{SE}=\mathrm{RL}>\mathrm{E} \\
8\end{array}$ & $\begin{array}{l}\mathrm{C}=\mathrm{E} 8=\mathrm{SE}=\mathrm{R} \\
\mathrm{L}\end{array}$ & $\mathrm{E} 8>\mathrm{SE}$ & $\begin{array}{l}\mathrm{E} 8>\mathrm{C}=\mathrm{SE}=\mathrm{R} \\
\mathrm{L}\end{array}$ \\
\hline & \multicolumn{8}{|c|}{$\mathrm{sO}_{2}(\mathrm{a})^{\mathrm{b}}$ (média \pm desvio-padrão) } \\
\hline $\mathrm{C}$ & $97,0 \pm 1,2$ & $98,0 \pm 0,7$ & $97,6 \pm 0,9$ & $97,8 \pm 0,8$ & $96,4 \pm 3,1$ & $97,6 \pm 0,6$ & $97,0 \pm 0,3$ & $97,6 \pm 1,3$ \\
\hline E8 & $97,0 \pm 0,0$ & $96,6 \pm 0,6$ & $96,2 \pm 1,1$ & $96,6 \pm 0,6$ & $95,8 \pm 1,6$ & $95,0 \pm 2,9$ & $95,2 \pm 2,6$ & $94,4 \pm 2,7$ \\
\hline $\mathrm{SE}$ & $97,5 \pm 1,0$ & $97,3 \pm 0,5$ & $96,5 \pm 1,0$ & $97,3 \pm 1,0$ & $95,0 \pm 1,4$ & $96,3 \pm 1,0$ & $96,3 \pm 1,0$ & $96,8 \pm 1,3$ \\
\hline \multirow[t]{2}{*}{ RL } & $97,6 \pm 0,6$ & $96,6 \pm 0,9$ & $95,8 \pm 1,6$ & $96,6 \pm 0,6$ & $97,4 \pm 1,8$ & $97,6 \pm 0,6$ & $97,0 \pm 0,7$ & $97,6 \pm 0,9$ \\
\hline & $\begin{array}{l}\mathrm{C}=\mathrm{E} 8=\mathrm{SE}=\mathrm{R} \\
\mathrm{L}\end{array}$ & $\mathrm{C}>\mathrm{E} 8=\mathrm{RL}$ & $\begin{array}{l}\mathrm{C}=\mathrm{E} 8=\mathrm{SE}=\mathrm{R} \\
\mathrm{L}\end{array}$ & $\begin{array}{l}\mathrm{C}=\mathrm{E} 8=\mathrm{SE}=\mathrm{R} \\
\mathrm{L}\end{array}$ & $\begin{array}{l}\mathrm{C}=\mathrm{E} 8=\mathrm{SE}=\mathrm{R} \\
\mathrm{L}\end{array}$ & $*$ & $*$ & $*$ \\
\hline
\end{tabular}

T0i-T72i: período de indução de compactação.

T12t-T48t: período de tratamento.

T72t: período final de tratamento.

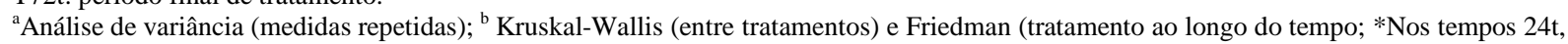
48t e 72f, os tratamentos não foram comparados, pois um animal do grupo SE morreu.

$\mathrm{C}=$ controle; $\mathrm{E} 8=$ solução isotônica poliônica enteral $\left(\mathrm{NaCl} 5 \mathrm{~g}+\mathrm{KCl} 1 \mathrm{~g}+\mathrm{NaHCO}_{3} 4 \mathrm{~g}+\mathrm{glicose} 5 \mathrm{~g}-\mathrm{qsq} 1000 \mathrm{ml}\right)$, dose de $8 \mathrm{~mL} \mathrm{~kg}^{-1} \mathrm{~h}^{-1} 48 \mathrm{~h}^{-1}$ na sonda nasogástrica; $\mathrm{SE}=$ solução isotônica intravenosa de Ringer lactato $\left(10 \mathrm{~mL} \mathrm{~kg}^{-1} \mathrm{~h}^{-1} 12 \mathrm{~h}^{-1}\right)$ associada à sene, dose de $20 \mathrm{mg}$ kg $\mathrm{R}^{-1}$, $\mathrm{RL}=$ solução isotônica de Ringer lactato $\left(16 \mathrm{~mL} \mathrm{~kg}^{-1} \mathrm{~h}^{-1} 12 \mathrm{~h}^{-1}\right)$.

\section{FONTES DE AQUISIÇÃO}

aPanacur pasta - Intervet S. A. - São Paulo-SP.

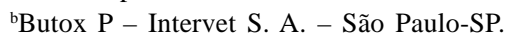

'Nutriage - Guabi S. A. - Além Paraíba-MG.

${ }^{\mathrm{d} C e n t a u r o ~-~ G u a b i ~ S . ~ A . ~-~ A l e ́ m ~ P a r a i ́ b a-M G . ~}$

éFresenius Kabi Brasil Ltda - Campinas-SP.

${ }^{\mathrm{f} B e c t o n}$ Dickinson Indústrias Cirúrgicas Ltda - Curitiba-PR.

'Becton Dickinson Indústrias Cirúrgicas Ltda - Curitiba-PR.

hLiquemine - Roche Químicos e Farmacêuticos.

${ }^{\mathrm{i} A B L 5}$ - Radiometer, Copenhagen.

\section{COMITÊ DE ÉTICA E BIOSSEGURANÇA}

O trabalho foi aprovado pela Comissão de Ética e Biossegurança da EV/UFMG.

\section{REFERÊNCIAS}

ANDREWS, J.M.; GRINDEM, C.B. Interpreting electrolyte, anion gap, and total carbon dioxide data. Vet Med, v.8, p.548553, 2000.
AINSWORTH, D.M.; BILLER, D.S. Respiratory system. In: REED, S.M.; BAYLY, W.M. Equine internal medicine. Philadelphia: Saunders, 1998. p.217-249.

ALVES, G.E.S. et al. Tratamento da compactação experimental do cólon maior em eqüinos: resultados de laboratório e exames bioquímicos. Arq Bras Med Vet Zootec, v.57, p.281-287, 2005.

BAILEY, J.E.; PABLO, L.S. Practical approach to acid-base disorders. Vet Clin North Am Small Animal Pract, v.28, p.645-662, 1998.

BREYER, J.; JACOBSON, R.H. Molecular mechanisms of diuretic agents. Annu Rev Med, v.141, p.265-270, 1990.

CARLSON, G.P. Fluid, electrolyte, and acid-base balance. In:______ Clinical biochemistry of domestic animal. 5.ed. San Diego: Academic, 1997. p.485-516.

CONOVER, W.J. Practical nonparametric statistics. New York: John Wiley \& Sons, 1980. 493p.

CORLEY, K.T.T.; MARR, C.M. Pathophysiology, assesment and treatment of acid-base disturbances in the horse. Equine Vet J, v.10, p.255-265, 1998. 
COSTA, A.P.D. Determinação dos efeitos da desidratação experimental nos equilíbrios hidroeletrolítico e ácidobase de eqüinos. 2003. 56f. Dissertação (Mestrado em Produção Animal) - Curso de Pós-graduação em Zootecnia, Universidade Estadual do Norte Fluminense.

DABAREINER, R.M.; WHITE, N.A. Large colon impaction: 147 cases (1985-1991). J Am Vet Med Assoc, v.206, p.679685, 1995.

DAVENPORT, H.W. ABC da química ácido básica do sangue. São Paulo: Atheneu, 1973. 127p.

DORAN, R. Field management of simple intestinal obstruction in horses. Comp Cont Educ Pract Vet, v.15, p.463-471, 1993.

FREESTONE, J.F. et al. Furosemide and sodium bicarbonateinduced alkalosis in the horse and response to oral $\mathrm{KCl}$ or $\mathrm{NaCl}$ therapy. Am J Vet Res, v.50, p.1334-1339, 1989.

GOMES, O.M. Interpretação clínica das alterações ácidobásicas e distúrbios da oxigenação. Belo Horizonte: Edicor, 1997. 254p.

GOSSET, K.A. et al. Contribuition of whole blood L-lactate, pyruvate, D-lactate, acetoacetate, and 3-hydroxybutirate concentrations to the plasma anion gap in horses with intestinal disordens. Am J Vet Res, v.48, p.72-75, 1987.

HOGMAN, C.F. et al. Effects on oxygen on red cells during storage at $4^{\circ} \mathrm{C}$. Vox Sang, v.51, p.27-44, 1986

JOHNSON, P.J. Electrolyte and acid-base disturbances in the horse. Vet Clin North Am Equine Pract., v.11, p.491-514, 1995.

KANEKO, J.R. et al. Clinical biochemistry of domestic animal. 5.ed. San Diego: Academic, 1997. 932p.

LARSEN, J. Acidbase and electrolyte balance in horses with various gastrointestinal disorders. In: EQUINE COLIC RESEARCH SYMPOSIUM, 5., 1994, Athens. Proceedings... Athens: University of Georgia, 1994. p.9.

MOORE, J.N. et al. A review of lactic acidosis with particular reference to the horse. J Equine Med Surg, v.1, p.96-105, 1977
MUIR, W.W.; de MORAIS, H.S.A. Acid-base balance: tradicional and modified approaches. In: Lumb \& Jones' Veterinary anesthesia. 3.ed. Baltimore: Williams \& Williams, 1996. p.558-571.

NAPPERT, G.; JOHNSON, P.J. Determination of the acid-base status in 50 horses admitted with colic between december1998 and may 1999. Can Vet J, v.42, p.703-707, 2001.

RIBEIRO FILHO, J.D.; ALVES, G.E.S. Compactação no intestino grosso de eqüinos: estudo comparativo de três protocolos de indução. Arq Bras Med Vet Zootec, v.54, p.370-375, 2002.

ROSE, R.J. et al. An evaluation of oral glucose-glycineelectrolyte solution for the treatment of experimentally induced dehydration in the horse. Vet Rec, v.119, p.522-525, 1986.

SAS. User's guide: statistics, version 5. Cary, 1985. 620p.

SPEIRS, V.C. The alimentary tract. In: Clinical examination of horses. Philadelphia: Saunders, 1997. p.261298.

SULLINS K.E. Disease of the large colon. In: WHITE, N.A. Equine acute abdomen. Philadelphia: Lea \& Febiger, 1990. p.375-391.

TERZI, R.G.G. Equilíbrio ácido-básico e transporte de oxigênio. São Paulo: Manole, 1992. 267p.

THOMASSIAN, A. Afecções do aparelho digestive. In: Enfermidades dos cavalos. 3.ed. São Paulo: Varela, 1997. p.325-518.

VALVERDE, A. et al. Sene vs polyethylene glycol for mechanical preparation the evening before elective colonic or retal resection. Arch Surg, v.134, p.514-519, 1999.

WARNER, A.E. Equine respiratory system: diseases of the lungs. In:___ Large animal internal medicine. 2.ed. St. Louis: Mosby, 2002. p.491-496. 